\title{
Diagnostic criteria for Menière's disease
}

Jose A. Lopez-Escamez ${ }^{\mathrm{a}, \mathrm{k}, *}$, John Carey ${ }^{\mathrm{b}}$, Won-Ho Chung ${ }^{\mathrm{c}}$, Joel A. Goebel ${ }^{\mathrm{d}}$, Måns Magnusson ${ }^{\mathrm{e}}$, Marco Mandala ${ }^{\mathrm{f}}$, David E. Newman-Toker ${ }^{\mathrm{g}}$, Michael Strupp ${ }^{\mathrm{h}}$, Mamoru Suzuki ${ }^{\mathrm{i}}$, Franco Trabalzini ${ }^{\mathrm{f}}$ and Alexandre Bisdorff $\mathrm{j}^{\mathrm{j}}$

${ }^{a}$ Otology and Neurotology Group CTS495, Department of Genomic Medicine, Centre for Genomics and Oncology Research - Pfizer/Universidad de Granada/Junta de Andalucía (GENyO), PTS, Granada, Spain

${ }^{\mathrm{b}}$ Department of Otolaryngology, Head and Neck Surgery, John Hopkins University School of Medicine, Baltimore, $M D, U S A$

${ }^{\mathrm{c}}$ Department of Otolaryngology, Head and Neck Surgery, Samsung Medical Center, Sungkyunkwan Univerisity School of Medicine, Seoul, Korea

${ }^{\mathrm{d}}$ Department of Otolaryngology, Head and Neck Surgery, Washington University School of Medicine, Saint Louis, MO, USA

${ }^{\mathrm{e}}$ Department of Otolaryngology, University of Lund, Lund, Sweden

${ }^{\mathrm{f}}$ Otology and Skull Base Department, Azienda Ospedaliera Universitaria Senese, Siena, Italy

${ }^{\mathrm{g}}$ Department of Neurology, John Hopkins University School of Medicine, Baltimore, MD, USA

${ }^{\mathrm{h}}$ Department of Neurology and German Center for Vertigo and Balance Disorders, Ludwig-Maximilians

University, Munich, Germany

${ }^{\mathrm{i}}$ Department of Otolaryngology, Tokyo Medical University, Tokyo, Japan

${ }^{\mathrm{j}}$ Department of Neurology, Centre Hospitalier Emile Mayrisch, Esch-sur-Alzette, Luxembourg

${ }^{\mathrm{k}}$ Department of Otolaryngology, Hospital de Poniente, El Ejido, Almeria, Spain

Received 2 March 2014

Accepted 10 Feburary 2015

Abstract. This paper presents diagnostic criteria for Menière's disease jointly formulated by the Classification Committee of the Bárány Society, The Japan Society for Equilibrium Research, the European Academy of Otology and Neurotology (EAONO), the Equilibrium Committee of the American Academy of Otolaryngology-Head and Neck Surgery (AAO-HNS) and the Korean Balance Society. The classification includes two categories: definite Menière's disease and probable Menière's disease. The diagnosis of definite Menière's disease is based on clinical criteria and requires the observation of an episodic vertigo syndrome associated with low- to medium-frequency sensorineural hearing loss and fluctuating aural symptoms (hearing, tinnitus and/or fullness) in the affected ear. Duration of vertigo episodes is limited to a period between 20 minutes and 12 hours. Probable Menière's disease is a broader concept defined by episodic vestibular symptoms (vertigo or dizziness) associated with fluctuating aural symptoms occurring in a period from 20 minutes to 24 hours.

Keywords: Vertigo, sensorineural hearing loss, tinnitus, vestibular disorders, Meniere disease

\footnotetext{
${ }^{*}$ Corresponding author: Jose A. Lopez-Escamez, Otology and Neurotology Group CTS495, GENyO, -Centre for Genomics and Oncological Research - Pfizer/Universidad de Granada/Junta de Andalucía, PTS Avda de la Ilustracion, 11418016 Granada, Spain. E-mail: antonio.lopezescamez@genyo.es.
}

\section{List of acronyms}

AAO-HNS - American Academy Otolaryngology Head and Neck Surgery

CCBS - Classification Committee of the Bárány Society

EAONO - European Academy of Otology and Neurotology

ICVD - International Classification of Vestibular Dis- 
orders

MD - Meniere's disease

SNHL - Sensorineural hearing loss

MRI - Magnetic resonance imaging

TIA - Transient ischemic attacks

\section{Introduction}

Menière's disease (MD) is a multifactorial disorder where the combined effect of genetics and environmental factors probably determine the onset of the disease. It is associated with the accumulation of endolymph in the cochlear duct and the vestibular organs in histopathological studies [1], although endolymphatic hydrops $(\mathrm{EH})$ per se does not explain all clinical features, including the progression of hearing loss or the frequency of attacks of vertigo [2]. Menière's disease is a clinical syndrome that consists of episodes of spontaneous vertigo usually associated with unilateral fluctuating sensorineural hearing loss (SNHL), tinnitus and aural fullness. Cochlear symptoms may also occur between vertigo episodes. The episodes of vertigo are usually much more common in the first years of the disease, but hearing loss and vestibular hypofunction show a great variability among patients, making the phenotyping of MD difficult. A taxonomy of MD subtypes is needed to help categorize individuals who manifest what may be incomplete phenotypes. Moreover, subsets of patients have been described who develop bilateral SNHL and other co-morbidities such as migraine, benign paroxysmal positional vertigo and systemic autoimmune diseases, further complicating the diagnosis and management in these cases.

The Bárány Society, which represents the international community of basic scientists, otolaryngologists, oto-neurologists, physical therapists and other experts committed to vestibular research, has set up a Classification Committee for an International Classification of Vestibular Disorders (ICVD). Individual disorders are defined by classification groups, which include otolaryngologists and neurologists from at least three continents. The ICVD has been organized in 3 levels: signs and symptoms, vestibular syndromes and diseases. As a first step and prerequisite for defining major syndromes and diseases of the ICVD, the Classification Committee of the Bárány Society (CCBS) has published a consensus on the definitions of vestibular symptoms [3]. Further, a consensus document of the Bárány Society and the International Headache Society was published to define the diagnostic criteria of vestibular migraine [4]. Current ongoing works include definition for vestibular signs such as nystagmus, benign paroxysmal positional vertigo, unilateral and bilateral vestibulopathy and vestibular paroxysmia.

Different efforts have been sought to define in the past a consensus diagnosis for MD. The Japanese Society for Equilibrium Research proposed clinical criteria for diagnosis of MD in 1974 (Appendix 1). The American Academy of Otolaryngology-Head and Neck Surgery (AAO-HNS) developed the guidelines for diagnosis and therapy evaluation of MD in 1972 and revised them in 1985 and 1995 [5]. These guidelines have been widely used in clinical research, and nearly all studies published since 1995 are based on these criteria (Appendix 2). Despite the great contribution of these guidelines to evaluate the effectiveness of medical and surgical treatments for MD, no biological marker for diagnosis or prognosis has been identified for MD so far, but an evolving understanding of MD and vestibular migraine - the most relevant differential diagnosis - points out the need to update these criteria. The clinical variability observed in MD makes it necessary to improve the precision to characterize clinical findings during and between the episodes of vertigo and the recognition of a familial history of hearing loss and episodic vertigo. These will lead to more homogeneous cohorts of patients to conduct clinical multicenter studies, including large-scale genomic studies. The EAONO had also established a working group to prepare European guidelines for diagnosis and treatment of MD.

The principal aim of this effort has been to develop international consensus diagnostic criteria for MD as part of the ongoing work of the CCBS to improve the quality of clinical data obtained from patients to facilitate collaborative studies.

\section{Methods}

This work forms part of an ongoing multi-year project to develop an ICVD, which uses a structured process for developing international consensus definitions for vestibular symptoms, syndromes, disorders, and diseases. This process, overseen by the CCBS, is based on expert, multi-disciplinary committees with international representation developing diagnostic criteria for subsequent comment and refinement prior to publication. These criteria are built on a critical appraisal of current best scientific evidence. A collaborative work among the Equilibrium Committee of 
the AAO-HNS, the Japan Society for Equilibrium Research, the EAONO, the Korean Balance Society and the Bárány Society has been established for joint development of diagnostic criteria for MD. All definitions are supported by notes, comments, and written discussion according to a template established by the CCBS for ICVD. MD criteria were developed iteratively over a four-year period (2010-14) through discussion, presentation, and refinement. Two diagnostic categories were considered: definite and probable MD. The primary adjustments to prior criteria were to clarify the nature of auditory symptoms and signs present for definite MD.

\section{Criteria for diagnosis of Menière's disease}

Previously used terms: Menière's syndrome, endolymphatic hydrops $(\mathrm{EH})$

\section{Definite $M D$}

A. Two or more spontaneous episodes of vertigo ${ }^{(1,2)}$, each lasting 20 minutes to 12 hours $^{(3)}$.

B. Audiometrically documented low- to mediumfrequency sensorineural hearing loss ${ }^{(4,5)}$ in one ear, defining the affected ear on at least one occasion before, during or after one of the episodes of vertigo ${ }^{(6,7)}$.

C. Fluctuating aural symptoms (hearing, tinnitus or fullness) in the affected ear ${ }^{(8)}$.

D. Not better accounted for by another vestibular $\operatorname{diagnosis}^{(9)}$.

Notes:

(1) Vertigo is the sensation of self-motion when no self-motion is occurring or the sensation of distorted self-motion during an otherwise normal head movement [3]. Episodic dizziness and unsteadiness are not considered as criteria to define MD, although patients with MD can report dizziness and unsteadiness at long term.

(2) Although most patients report entirely spontaneous spells of vertigo, some patients identify dietary triggers, such as excessive consumption of sodium or caffeine. Some patients can experience episodes of vertigo lasting seconds to minutes triggered by high intensity and lowfrequency sound (Tullio phenomenon) and by changes in pressure. These episodes tend to occur later in the disease, perhaps as a result of advanced hydrops bringing the membranous labyrinth in close proximity to the stapes footplate.
(3) The duration of the episodes is defined by the time that the patient has to be on rest and cannot move. It may last less than 20 minutes or more than 12 hours, but neither is a common finding, and other disorders should be considered when such durations are noted [6]. When short episodes occur they are usually spontaneous. Brief episodes triggered by positional changes of the head should suggest other causes such as BPPV. The duration of an episode may be difficult to define as patients may have residual symptoms after an episode.

(4) The affected ear is determined by hearing loss defined in criteria B. Low-frequency SNHL is defined as increases in pure tone thresholds for bone-conducted sound that are higher (i.e., worse) in the affected ear than the contralateral ear by at least $30 \mathrm{~dB} H L$ at each of two contiguous frequencies below $2000 \mathrm{~Hz}$. In cases of bilateral low-frequency SNHL, the absolute thresholds for bone-conducted sound must be $35 \mathrm{~dB}$ HL or higher at each of two contiguous frequencies below $2000 \mathrm{~Hz}$. If multiple audiograms are available, demonstration of recovery of low-frequency SNHL at some point in time further supports the diagnosis of MD. Bilateral synchronous SNHL (symmetric or asymmetric) can occur in some patients [7.-9], although such a pattern should raise concern for the possibility of autoimmune inner ear disease [10] and when progressing slowly over years, may favor migraine as an alternate explanation for the vertigo episodes or a comorbid condition [11]. Bilateral low-frequency SNHL can also been observed in early stages of non-syndromic progressive deafness (DFNA6/14) due to mutations in the WFS1 gene, but vertigo attacks have not been associated with this group of mutations [12]. SNHL in MD can also involve middle and high frequencies after several episodes of vertigo, leading to pan-tonal hearing loss.

(5) Non-simultaneous onset of vertigo and hearing loss. Sensorineural hearing loss may antedate the onset of vertigo episodes by several months or years. This clinical variant has been called "delayed hydrops" [13 14], but the preferred term should be delayed $M D$, since $\mathrm{EH}$ is a pathological finding. Episodic vertigo may precede the onset of hearing loss by several weeks or months, but tinnitus or aural fullness is usually associated with the first episode of vertigo. 
(6) A temporal association between hearing loss and the vertigo episode is sometimes observed by the patient, generally as a change in hearing within 24 hours of the vertigo episode. Hearing loss typically fluctuates spontaneously in the first few years of the disease. After repeated attacks, the hearing loss may progress and become permanent, and the episodes of vertigo may likewise no longer be related with the aural symptoms.

(7) Attacks of sudden loss of vestibulo-spinal reflexes resulting in sudden falls or less often latero-pulsion and lasting seconds or, rarely, a few minutes can occur (so called drop vestibular attacks, otolithic crises or Tumarkin's otolithic crises) [15[16].

(8) An increase in tinnitus intensity or aural fullness in the affected ear is often associated with the episode of vertigo in the first years. Tinnitus may be persistent once the hearing loss has become permanent.

(9) The differential diagnosis should include transient ischemic attack, vestibular migraine, vestibular paroxysmia, recurrent unilateral vestibulopathy and other vestibular disorders. MRI may be required to exclude vestibular schwannoma or endolymphatic sac tumor. Migraine, benign paroxysmal positional vertigo and some forms of systemic autoimmune conditions are considered co-morbidities and do not explain the diagnosis of MD. See Comments for discussion of specific differential diagnoses.

\section{Probable MD}

A. Two or more episodes of vertigo or dizziness, each lasting 20 minutes to 24 hours.

B. Fluctuating aural symptoms (hearing, tinnitus or fullness) in the affected ear ${ }^{(1)}$.

D. Not better accounted for by another vestibular $\operatorname{diagnosis}^{(2)}$.

Notes:

(1) Fluctuating symptoms must be reported during the vertigo episode. Sensorineural hearing loss is usually found, but conductive or mixed hearing loss may be also observed in the first years of the disease. An increase in tinnitus intensity or aural fullness in the affected ear is usually associated with the episode of vertigo in the first years.
Table 1

Differential diagnosis of Menière's disease

Autosomal dominant sensorineural hearing loss type 9 (DFNA9)
caused by COCH gene
Autosomal dominant sensorineural hearing loss type 6/14
(DFNA6/14) caused by WSF1 gene
Autoimmune inner ear disease
Cerebrovascular disease (stroke/TIA in the vertebrobasiliar sys-
tem/bleeding)
Cogan's syndrome. Some cases may have recurrences.
Endolymphatic sac tumor
Meningiomas and other masses of the cerebellopontine angle
Neuroborreliosis
Otosyphilis
Susac syndrome
Third window syndromes (Perilymph fistula, canal dehiscence, en-
larged vestibular aqueduct)
Vestibular migraine
Vestibular paroxysmia (neurovascular compression syndrome)
Vestibular schwannoma
Vogt-Koyanagi-Harada syndrome

(2) The differential diagnosis should include transient ischemic attack, vestibular migraine and other vestibular disorders. MRI may be required to exclude vestibular schwannoma or endolymphatic sac tumor. Migraine, benign paroxysmal positional vertigo and some forms of systemic autoimmune conditions are considered co-morbidities and do not explain the clinical syndrome of MD. See Comments for discussion of specific differential diagnoses.

\section{Comments}

\subsection{Epidemiology}

The prevalence of MD is approximately 34-190 per 100.000 [17.19]. The age of onset ranges from the third to the seventh decades of life with a small female predominance. The odds of MD are greater for older subjects (adjusted OR per 10-year increase: 1.5), white ethnicity (OR 1.7) and severe obesity (OR 1.7) [19]. Furthermore, MD is associated with several comorbid conditions such as arthritis (OR 1.8), psoriasis (OR 1.8), gastroesophageal reflux disease (OR 1.5), irritable bowel syndrome (OR 2.1) and migraine (OR 2.0) [19]. MD is characterized by a variable course, and the development of cochlear and vestibular symptoms may take years in individual patients [20]. A list of disorders mimicking MD is presented in Table 1 . 


\subsection{Endolymphatic hydrops $(\mathrm{EH})$}

Histopathological studies in human temporal bones have found $\mathrm{EH}$ in most patients with MD [1/2]. The recent development of $3 \mathrm{~T}$ MRI with gadolinium chelate has allowed the visualization of $\mathrm{EH}$ in $93 \%$ of ears with symptoms attributable to $\mathrm{MD}$, but also in $65 \%$ of asymptomatic contralateral ears [20]. In addition, electrocochleography is a neurophysiological test that correlates with $\mathrm{EH}$ in the cochlea; however, the fluctuating symptoms limit their applicability as diagnostic tool in the early course of MD [21].

\subsection{Familial MD}

Familial MD should be considered if at least one other relative (first or second degree) fulfills all the criteria of definite or probable MD [22]. Familial MD is observed in $8 \%-9 \%$ of sporadic cases in populations of European descent [22|23]. It has been described in Caucasians from United Kingdom [24], Brazil [25], Sweden [26], Finland [2327], Germany [28] and Spain [23]. Although most families described have an autosomal dominant pattern of inheritance, familial MD shows genetic heterogeneity, and mitochondrial and recessive inheritance patterns are also observed in some families [22]. Mutations in DTNA and FAM136A genes have been described in familial MD [29]. A family history of SNHL, migraine or recurrent vertigo in several members of the family would justify a familial investigation of these patients to confirm if any of them fulfill diagnostic criteria for MD.

\subsection{Overlap with vestibular migraine}

Some studies suggest that migraine is more common in patients with MD than in healthy controls [30 31]. Patients with features of both MD and vestibular migraine have been repeatedly reported [32]. In fact, migraine and MD can be inherited as a symptom cluster [33]. Fluctuating hearing loss, tinnitus and aural pressure may occur in vestibular migraine, but SNHL does not typically progress to severe hearing loss over the years in migraine. When hearing loss develops in vestibular migraine, it is often bilateral [32] whereas involvement of both ears from the onset is rare in MD [34]. Although migraine headache and phonophobia may be present during MD attacks, the pathophysiological relationship between vestibular migraine and MD is unclear. Some authors have suggested a common pathophysiology, perhaps linked to an ion channel dysfunction [35] and endolymphatic hydrops has been reported in patients with vestibular migraine and auditory symptoms [36].

\subsection{Autoimmune inner ear disease}

Autoimmune inner ear disease (AIED) is a syndrome of rapidly progressive, often fluctuating, bilateral SNHL over a period of days to months. Vestibular symptoms may be present in almost $50 \%$ of patients, and a systemic autoimmune disease coexists in $15 \%-30 \%$ of patients [37]. In some cases, AIED begins as sudden SNHL in one ear progressing rapidly to the second ear with tinnitus and balance symptoms, which may resemble MD [38]. Rare cases of otologic syphilis and neuroborreliosis can present similarly.

\subsection{Transient ischemic attack and stroke}

Combined audio-vestibular symptoms are also a hallmark of transient ischemic attack (TIA) and stroke, since the blood supply to the inner ear derives from the vertebro-basilar circulation [39]. Such symptoms typically result from ischemia in the anterior inferior cerebellar artery (AICA) territory [40[41], since the internal auditory artery (IAA) most often arises as a branch of the AICA. However, the IAA can arise directly from the basilar artery in $15 \%-20 \%$ of individuals [42], so mixed vestibular and auditory symptoms may be a harbinger of basilar artery occlusion.

Patients may present initially with transient or episodic symptoms (TIA) that culminate in stroke with acute permanent hearing loss, permanent vestibular loss, or both [43]. Transient dizziness, hearing loss, and/or tinnitus may occur as a premonitory TIA preceding acute audio-vestibular loss due to AICA infarction in up to $42 \%$ of cases. [40]43] Although neurologic symptoms such as facial palsy or hemiparesis can occur in patients with basilar strokes, some patients with partial syndromes can manifest with isolated audio-vestibular symptoms almost indistinguishable from MD [44]. Furthermore, TIA symptoms can culminate in isolated labyrinthine infarction (without cerebellar or brainstem involvement) [45].

Most strokes after TIA occur within 90 days [45], but isolated vestibular symptoms have been reported for up to two years or more preceding stroke. Therefore, great care should be taken in patients with symptoms that are very recent in total illness duration $(<$ 3 months- 6 months, especially $<30$ days), particularly if the total number of episodes is relatively small (e.g., $<5$ ) or has followed an escalating temporal profile of episode frequency ("crescendo TIA") [44]. Care should also be taken in patients with known vascular risk factors (including older age at first onset) and those 
presenting to acute care settings such as the emergency department. When clinically-important doubt exists, appropriate diagnostic testing for stroke and TIA (e.g., MRI with magnetic resonance angiography) should be considered. The first episode of MD may not differ from a TIA, and sudden SNHL associated with vertigo may be caused by a labyrinthine infarction.

\section{Acknowledgments}

The Barany Society, the EAONO and Neuro+, Berlin have supported the working meetings to develop a consensus definition for MD. The authors are thankful for all the comments and suggestions received from the vestibular community worldwide.

\section{References}

[1] S.D. Rauch, S.N. Merchant and B.A. Thedinger, Menière's syndrome and endolymphatic hydrops: Double-blind temporal bone study, Ann Otol Rhinol Laryngol 98 (1989), 873-883.

[2] S.N. Merchant, J.C. Adams and J.B. Nadol, Pathophysiology of Menière' syndrome are symptoms caused by endolymphatic hydrops? Otol Neurotol 26 (2005), 74-81.

[3] A. Bisdorff, M. von Brevern, T. Lempert and D. NewmanToker (on behalf of the committee for the classification of vestibular disorders of the Bárány society), classification of vestibular symptoms: Towards an international classification of vestibular disorders, J Vest Res 19 (2009), 1-13.

[4] T. Lempert, J. Olesen, J. Furman, J. Waterston, B. Seemungal, J. Carey et al., Vestibular migraine: Diagnostic criteria, $J$ Vestib Res 22 (2012), 167-172.

[5] Committee on hearing and equilibrium, guidelines for the diagnosis and evaluation of therapy in Menière's disease, Otolaryngol Head Neck Surg 113 (1995), 181-185.

[6] H. Perez-Garrigues, J.A. Lopez-Escamez, P. Perez, R. Sanz, M. Orts, J. Marco, R. Barona, M.C. Tapia, I. Aran, C. Cenjor, N. Perez, C. Morera and R. Ramirez, Time course of episodes of definitive vertigo in Menière's disease, Arch Otolaryngol Head Neck Surg 134 (2008), 1149-1154.

[7] S. Nabi and L.S. Parnes, Bilateral Ménière's disease, Curr Opin Otolaryngol Head Neck Surg 17 (2009), 356-362.

[8] J.A. Lopez-Escamez, D. Viciana and P. Garrido-Fernandez, Impact of bilaterality and headache on health-related quality of life in Menière's disease, Ann Otol Rhinol Laryngol 118 (2009), 409-416.

[9] A. Belinchon, H. Perez-Garrigues, J.M. Tenias and A. Lopez, Hearing assessment in Menière's disease, Laryngoscope 121 (2011), 622-626.

[10] S. Pathak, L.J. Hatam, V. Bonagura and A. Vambutas, Innate immune recognition of molds and homology to the inner ear protein, cochlin, in: Patients with Autoimmune Inner Ear Disease, J Clin immunol 33 (2013), 1204-1215.

[11] Y.H. Cha, J. Brodsky, G. Ishiyama, C. Sabatti and R.W. Baloh, The relevance of migraine in patients with Menière's disease, Acta Otolaryngol 127 (2007), 1241-1245.
[12] R.J. Pennings, S.J. Bom, K. Cryns, K. Flothmann, P.L. Huygen, H. Kremer, G. Van Camp and C.W. Cremers, Progression of Low-Frequency Hearing Loss Sensorineural Hearing Loss (DFNA6/14-WFS1) Arch Otolaryngol Head Neck Surg 129 (2003), 421-426.

[13] H.F. Schuknecht, Y. Suzuka and C. Zimmermann, Delayed endolymphatic hydrops and its relationship to Meniére's disease, Ann Otol Rhinol Laryngol 99 (1990), 843-853.

[14] T. Kamei, Delayed endolymphatic hydrops as a clinical entity, Int Tinnitus J 10 (2004), 137-143.

[15] E. Kentala, M. Havia and I. Pyykkö, Short-lasting drop attacks in Menière's disease, Otolaryngol Head Neck Surg 124 (2001), 526-530.

[16] N. Perez-Fernandez, L. Montes-Jovellar, J. Cervera-Paz and E. Domenech-Vadillo, Auditory and vestibular assessment of patients with Ménière's disease who suffer Tumarkin attacks, Audiol Neurootol 15 (2010), 399-406.

[17] H. Shojaku, Y. Watanabe, M. Fujisaka et al., Epidemiologic characteristics of definite Menière's disease in Japan, a longterm survey of toyama and niigata prefectures, ORL J Otorhinolaryngol Relat Spec 67(5) (2005), 305-309.

[18] T.H. Alexander and J.P. Harris, Epidemiology of Menière's syndrome, Otolaryngol Clin N Am 43 (2010), 965-970.

[19] J.S. Tyrrell, D.J.D. Whinney, O.C. Ukoumunne, L.E. Fleming and N.J. Osborne, Prevalence, associated factors and comorbid conditions for Ménière's disease, Ear and Hearing 35 (2014), e162-e169.

[20] I. Pyykkő, T. Nakashima, T. Yoshida, J. Zou and S. Naganawa, Meniere's disease: A reappraisal supported by a variable latency of symptoms and the MRI visualisation of endolymphatic hydrops, BMJ Open 3(2) (2013), e001555.

[21] P. Lamounier, D.A. Gobbo, T.S.A. de Souza, C.A.C.P. de Oliveira and F. Bahmad, Electrocochleography for Ménière's disease: Is it reliable? Braz J Otorhinolaryngol 80 (2014), 527-532.

[22] T. Requena, J.M. Espinosa-Sanchez, S. Cabrera, G. Trinidad, A. Soto-Varela, S. Santos-Perez et al., Familial clustering and genetic heterogeneity in Menière's disease, Clinical Genetics 85 (2014), 245-252.

[23] E. Hietikko, J. Kotimäki, M. Sorri and M. Männikkö, High incidence of Menière-like symptoms in relatives of Menière patients in the areas of oulu university hospital and kainuu central hospital in finland, Eur J Med Genet 56 (2013), 179185.

[24] A.W. Morrison, M.E. Bailey and G.A. Morrison, Familial Ménière's disease: Clinical and genetic aspects, J Laryngol Otol 123 (2009), 29-37.

[25] C.A. Oliveira, C.I. Messias and I. Ferrari, Occurrence of familial Ménière's syndrome and migraine in Brasilia, Ann Otol Rhinol Laryngol 111 (2002), 229-236.

[26] D. Gabrikova, C. Frykholm, U. Friberg, S. Lahsaee, M. Entesarian, N. Dahl and J. Klar, Familiar Menière's disease restricted to $1.48 \mathrm{Mb}$ on chromosome $12 \mathrm{p} 12.3$ by allelic and haplotype association, J Hum Genet 55 (2010), 834-837.

[27] E. Hietikko, J. Kotimäki, E. Kentala, T. Klockars, M. Sorri and M. Männikkö, Finnish familial Menière disease is not linked to chromosome $12 \mathrm{p} 12.3$, and anticipation and cosegregation with migraine are not common findings, Genet Med $\mathbf{1 3}$ (2011), 415-420.

[28] D. Arweiler-Harbeck, B. Horsthemke, K. Jahnke and H.C. Hennies, Genetic aspects of familial Menière's disease, Otol Neurotol 32 (2011), 695-700.

[29] T. Requena, S. Cabrera, C. Martín-Sierra, S.D. Price, A. Lysakowski and J.A. Lopez-Escamez, Identification of two 
novel mutations in FAM136A and DTNA genes in autosomaldominant familial Meniere's disease, Hum Mol Genet 24 (2015), 1119-1126.

[30] A. Radtke, T. Lempert, M.A. Gresty, G.B. Brookes, A.M. Bronstein and H. Neuhauser, Migraine and Menière's disease: Is there a link? Neurology 59 (2002), 1700-1704.

[31] Q. Gopen, E. Virre and J. Anderson, Epidemiologic study to explore links between Ménière syndrome and migraine headache, Ear Nose Throat J 88 (2009), 1200-1204.

[32] A. Radtke, H. Neuhauser, M. von Brevern, T. Hottenrott and T. Lempert, Vestibular migraine-validity of clinical diagnostic criteria, Cephalalgia 31 (2011), 906-913.

[33] Y.H. Cha, M.J. Kane and R.W. Baloh, Familial clustering of migraine, episodic vertigo and Menière's disease, Otol Neurotol 29 (2008), 93-96.

[34] D. Huppert, M. Strupp and T. Brandt, Long-term course of Menière's disease revisited, Acta Otolaryngol 130 (2010), 644-651.

[35] P. Gates, Hypothesis: Could Menière's disease be a channelopathy? Intern Med J 35 (2005), 488-489.

[36] R. Gürkov, C. Kantner, M. Strupp, W. Flatz, E. Krause and B. Ertl-Wagner, Endolymphatic hydrops in patients with vestibular migraine and auditory symptoms, Eur Arch Otorhinolaryngol 271 (2014), 2661-2667.

[37] R. Bovo, C. Aimoni and A. Martini, Immune-mediated inner ear disease, Acta Otolaryngol 126 (2006), 1012-1021.

[38] B.F. McCabe, Autoimmune sensorineural hearing loss, Ann Otol Rhinol Laryngol 88 (1979), 585-589.

[39] J.G. Oas and R.W. Baloh, Vertigo and the anterior inferior cerebellar artery syndrome, Neurology 42 (1992), 2274-2279.

[40] H. Lee, S.I. Sohn, D.K. Jung, Y.W. Cho, J.G. Lim, S.D. Yi, S.R. Lee, C.H. Sohn and R.W. Baloh, Sudden deafness and anterior inferior cerebellar artery infarction, Stroke $\mathbf{3 3}$ (2002), 2807-2812.

[41] H. Lee and R.W. Baloh, Sudden deafness in vertebrobasilar ischemia: Clinical features, vascular topographical patterns and long-term outcome, J Neurol Sci 228 (2005), 99-104.

[42] R. Häusler and R.A. Levine, Auditory dysfunction in stroke, Acta Otolaryngol 120 (2000), 689-703.

[43] H. Lee, Neuro-otological aspects of cerebellar stroke syndrome, J Clin Neurol 5 (2009), 65-73.

[44] H. Lee and Y.W. Cho, Auditory disturbance as a prodrome of anterior inferior cerebellar artery infarction, J Neurol Neurosurg Psychiatry 74 (2003), 1644-1648.

[45] P.M. Rothwell, A. Buchan and S.C. Johnston, Recent advances in management of transient ischemic attacks and minor ischaemic strokes, Lancet Neurol 5 (2006), 323-331.

\section{Appendix 1. Criteria for diagnosis of Menière's disease. Menière's disease Research Committee of Japan. 1974.}

\section{Conditions}

1. Repeated attack of whirling vertigo.

Dizzy spells without specific cause which are accompanied by nausea or vomiting lasting several minutes to several hours. There may be some episodes of nonwhirling dizziness included in the series of whirling vertigo; mixed types of sponta- neous nystagmus (horizontal and rotatory is observed in most cases during the attacks; in cases with a single first attack, differential diagnosis with sudden SNHL is specially important.

2. Fluctuating cochlear symptoms.

Tinnitus and hearing loss often show fluctuation synchronous with the vertiginous attacks; many patients complain of fullness in the ear and hypersensitivity to intense sound in the affected ear. The hearing tests reveal a marked fluctuation of the threshold of hearing in the low and middle tone range, recruitment of loudness will be observed; usually only one ear is affected, however, bilateral involvement is not rare.

3. Exclusion of central nervous system involvement, VIIIth nerve tumor and other cochleovestibular diseases.

To exclude these other disorders, a through history, neurological examination and specific clinical examination including equilibrium function tests, audiological tests must be performed sometimes it is necessary to follow the patient's course in order to obtain the required chronological information necessary for establishing the correct diagnosis.

\section{Diagnostic criteria}

I. Definite Menière disease: Condition 1-3.

II. Suspicious or uncertain Menière disease 1 and 3 or 2 and 3.

\section{Appendix 2. Menière's disease diagnostic scale. committee on hearing and equilibrium american academy otolaryngology-head neck surgery. 1995.}

Certain Menière's disease. Definitive Menière's disease plus histopathological confirmation

Definite Menière's disease. Two or more definitve spontaneous episodes of vertigo lasting 20 minutes or longer, audiometrically documented hearing loss on at least one occasion, tinnitus or aural fullness in the treated ear. Other causes excluded.

Probable Menière's disease. One definitive episode of vertigo, audiometrically documented hearing loss on at lest one occasion, tinnitus or aural fullness in the treated ear. Other causes excluded.

Possible Menière's disease. Episodic vertigo without documented hearing loss or sensorineural hearing loss fluctuating or fixed with disequilibrium but without definitive episodes. Other causes excluded. 